\title{
Third party funders must come to the party in response to oral health PPE, the government must address issues of lack of water and PPE price escalation
}

SADJ June 2020, Vol. 75 No. 5 p233 - p234

KC Makhubele

South African Oral Health Care $(\mathrm{OHC})$ providers, just like all over the world, are considered essential services and are tasked with addressing the challenges of providing treatment during and after the COVID-19 pandemic.

$\mathrm{OHC}$ is an essential need, which, if left unattended could lead to crises that are life threatening or even fatal. Thus, continued care is vital to safeguard the (oral and systemic) health of our patients, prevent serious complications and sustain the profession.

The fights against the pandemic if further compounded by factors such as: the lack of water in rural areas and the escalation of PPE cost.

SADA has noted with great concern that life-saving protective equipment is running critically low in facilities across South Africa, and the world, due to increased demand created by the COVID-19 pandemic. The PPE stock continues to be affected in light of export restrictions, increased costs and disruptions in logistics.

Many of our $\mathrm{OHC}$ have not been able to procures the necessary PPE. SADA is becoming increasingly frustrated that as well as its members not being to procure PPE, suppliers are unable to give answers as to when PPE stocks would be available.

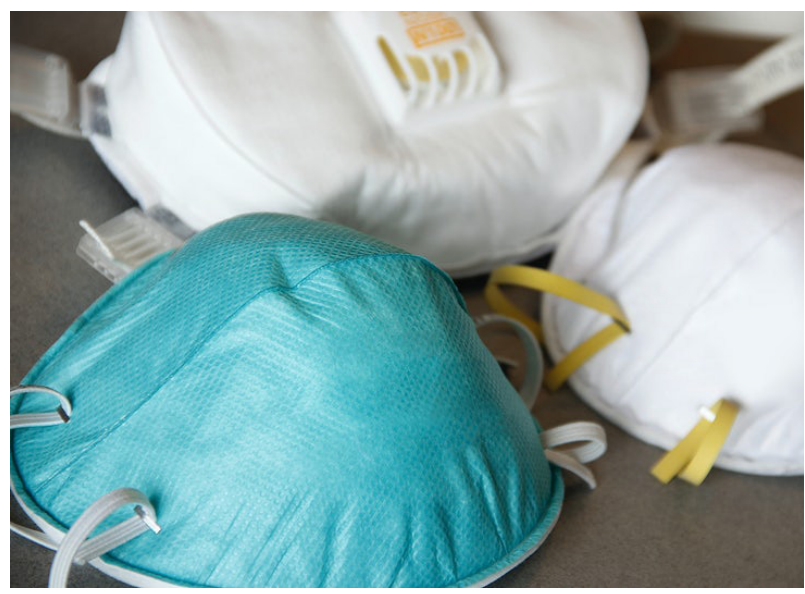

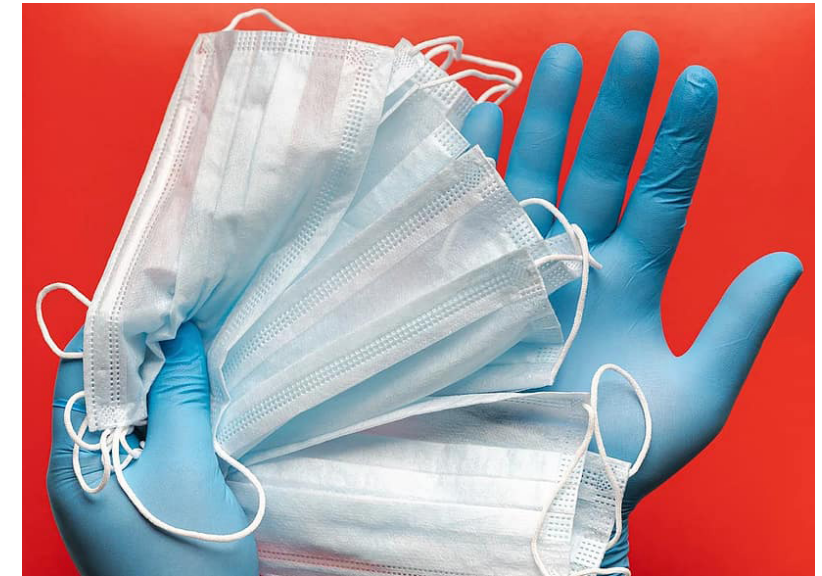

Since COVID-19 we have noted a more than 1000\% increase in costs for required personal protective equipment since the deadly virus started its rampage in the South Africa. This increase continues. The increase is attributes to supply-and-demand factors, as well as the increased number of items mandated for safety, and the call for frequent changes. The government seems to be heading the call and we hope to see the escalation curtailed.

We equally also concerned about the lack of water in rural settlement and health facilities across the country. These areas are the most vulnerable and may become key centers on the spread of the coronavirus. We however remain hopeful that Minister of Human Settlements, Water and Sanitation, Lindiwe Sisulu, will fulfill her promise that that water provisions, such as tanks and stand pipes, for rural and informal areas would be drastically increased as a means to further fight the pandemic.

The case for infection control measures to be provided for by third party funders

The very close proximity of the $\mathrm{OHC}$ providers to their patients as well as the massive generation of aerosols during routine and emergency procedures increases the risk of COVID-19 contamination and cross infection. 
For years, OHC workers have adopted, extensive precautions against numerous blood borne viral illnesses such as HIV and hepatitis, and fungal and bacterial challenges. The current trends in infection control and use of personal protective equipment (PPE) have been adequate to meet this challenge. However, the advent of COVID-19, with a different vector of infection, has made our current cross infection control strategies entirely inadequate.

The production of aerosols leads to extensive contamination of our surgeries, surgical scrubs and our PPE. This significant contamination requires that we efficiently disinfect our surgeries and appropriately disinfect or dispose of some of the PPE.

As a response to the current crisis, the SADA protocols committee developed a document that provides scientific guidelines for safe practice for all $\mathrm{OHC}$ workers and especially for their patients.

We refer to Dental Clinical Protocol in Response to the COVID-19 Pandemic 2020: A South African Perspective, which has been widely adopted by all $\mathrm{OHC}$ workers in South Africa, academia and the HPCSA.

In addition to the standard PPE required, SADA advocates for the use of Rubber Dam as an Essential Protection Measure. Not only is the use of rubber dam considered critical in the reduction of infectious pathogen transmission, but scientific reports highlight why the use of rubber dam should be considered mandatory for all routine endodontic and restorative dental procedures.

The advantages include but not limited to:

- The prevention of cross contamination.

- Protection of both operating staff as well as the patient.

- Improved effectivity and longevity of dental treatment.

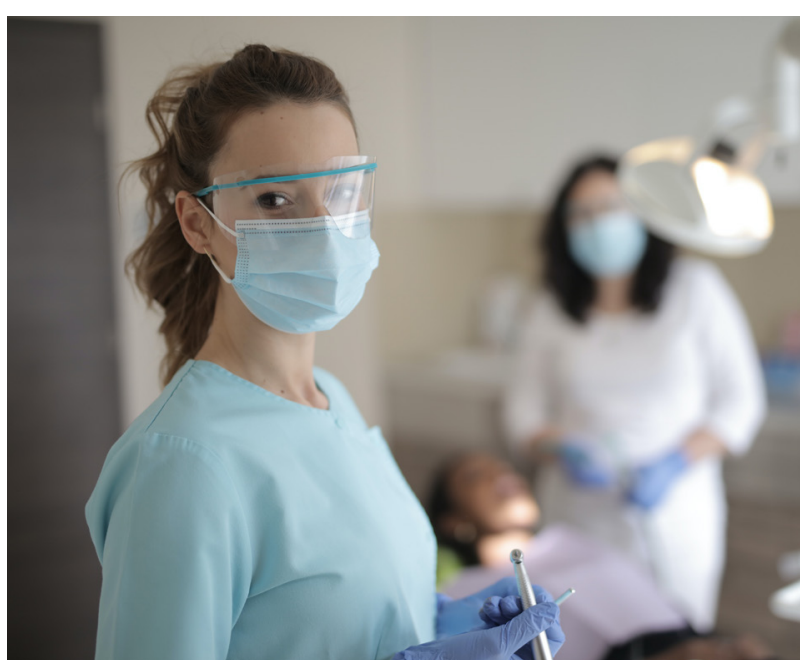

The protective nature of rubber dam for both patient and operating staff in conjunction with its many clinical advantages is largely underestimated and undervalued and should therefore be classified as essential and standard protocol for dental procedures.
This requires extra-ordinary measures in the dental environment to protect patients, employees and practitioners, and they must be carried out in accordance with the latest international and SADA guidelines and protocols. The use of PPE, routine use of rubber dam (for both endodontic and restorative procedures) as well as thorough disinfection and sterilisation procedures cannot be relaxed until a vaccine or cure has been developed or herd immunity has been achieved.

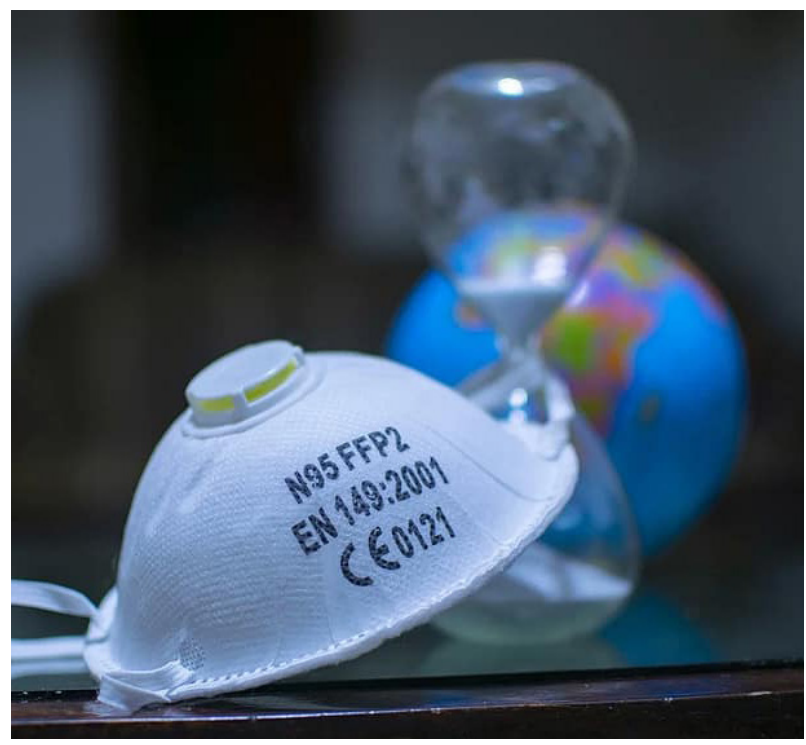

These measures result in a significant increase in the costs of providing of dental services, which are not catered for in the current reimbursement system, and many practices will be unable to fund them without support. However, this cost is small compared to that of losing a life. Our priority is the safety and wellbeing of our patients and their families, our staff and their families and ourselves and our families.

SADA is currently engaged with third party funders to ensure they support the infection control measures adopted by the profession. 